\title{
UTILIZATION OF LARGE SCALE SURFACE MODELS FOR DETAILED VISIBILITY ANALYSES
}

\author{
Jan Caha ${ }^{\mathrm{a}, *}$, Michal Kačmařík ${ }^{\mathrm{b}}$ \\ ${ }^{\text {a }}$ Department of Regional Development, Mendel University in Brno, Zemědělská 1, 61300 Brno, Czech Republic - \\ jan.caha@mendelu.cz \\ ${ }^{\mathrm{b}}$ Institute of Geoinformatics, VŠB-Technical University of Ostrava, 17. listopadu 15, 70833 Ostrava-Poruba, Czech Republic - \\ michal.kacmarik@vsb.cz
}

Commission II

KEY WORDS: analysis of visibility, unmanned air vehicle, 3d point cloud, extended viewshed, viewshed

\begin{abstract}
:
This article demonstrates utilization of large scale surface models with small spatial resolution and high accuracy, acquired from Unmanned Aerial Vehicle scanning, for visibility analyses. The importance of large scale data for visibility analyses on the local scale, where the detail of the surface model is the most defining factor, is described. The focus is not only the classic Boolean visibility, that is usually determined within GIS, but also on so called extended viewsheds that aims to provide more information about visibility. The case study with examples of visibility analyses was performed on river Opava, near the Ostrava city (Czech Republic). The multiple Boolean viewshed analysis and global horizon viewshed were calculated to determine most prominent features and visibility barriers of the surface. Besides that, the extended viewshed showing angle difference above the local horizon, which describes angular height of the target area above the barrier, is shown. The case study proved that large scale models are appropriate data source for visibility analyses on local level. The discussion summarizes possible future applications and further development directions of visibility analyses.
\end{abstract}

\section{INTRODUCTION}

The dependency of viewshed analysis results on quality of the surface model is a well-documented phenomenon (Maloy and Dean, 2001). The precision of the visibility assessment rises with increasing amounts of information about the surface. The first research in the field of visibility was often done purely on terrain models with cell size often bigger than 10 meters (e.g. Lee, 1991) the obtained results were unsurprisingly not precise, with level of agreement between predicted and field surveyed viewsheds only slightly higher than 50 percent (Maloy and Dean, 2001). Later studies proved that it is necessary to have data with significantly smaller spatial resolution of maximal cell size 5 meters and with elevation accuracy lower than 1 meter (Klouček et al., 2015) to obtain reliable visibility predictions. However, even data with such precision and spatial resolution are suitable rather for visibility analyses on larger areas.

We can divide the visibility analyses into two main types - local and regional. The regional analyses are performed on larger areas and focus on visibility of significant features of the landscape such as mountain peaks, man-made structures, cities etc. For this type of analyses the model of the surface does not have to be extremely detailed, as the outcomes of the analysis need to cover mainly the general visibility of the target. The local analysis of visibility on the other hand focuses on visibility of smaller targets, like statues, column shrines, signs or billboards and other important landmarks from their relatively close surroundings. This type of visibility analysis requires much more precise and detailed surface models as the outcomes should describe the visibility in significantly greater detail (Chmielewski and Tompalski, 2017). As the target object and the area of interest get smaller the precision, resolution and level of detail of the surface model need to be both finer and more accurate.
As noted by Remondino et al (2011) the UAVs (Unmanned Aerial Vehicle) are optimal source of data if the area of interest is not very large (hectares or first squared kilometres). The scanning from low heights allow the photogrammetrically 3D data to have unmatched precision especially in capturing details of buildings and vegetation (Dandois and Ellis, 2013; Tomaštík et al., 2017). If the conditions are convenient, the surfaces derived from UAV scanning can cover even areas under partial tree canopy (Tomaštík et al., 2017) which can be used to create full 3D models of the vegetation instead of 2.5D surface which are much more common in geoinformatics. Due to this fact, the photogrammetrically derived surfaces can be extremely good data source for local visibility analysis.

One of the main reasons for using UAV is the ability to collect data quickly and cheaply in comparison with other methods of surface data collection. Due to the fact that vegetation plays an important role in local visibility analysis it is usually fitting to have the same dataset in different seasons as the amount and density of vegetation changes considerably through the year. In such situations low time and cost demands of data collection with the utilization of UAV are significant pros.

The photogrammetrically derived surfaces are suitable not only for classic Boolean visibility analyses but also for determination of so called extended viewsheds (sometimes called visibility indexes), which aims to provide more information about the visibility (Fisher, 1996b). There are many possible extended viewsheds that can be calculated but amongst the most important are horizon viewshed (Fisher, 1996a) and angle elevation above local horizon (Caha and Rášová, 2015). The utilization of high precision surface models leads to more revealing results.

\footnotetext{
* Corresponding author
} 
In this contribution we will describe the utilization of cloud point data for large scale detailed visibility analysis. Both Boolean and extended viewsheds (Fisher, 1996; Caha and Rášová, 2015) are calculated for selected observation points. The research was conducted on a few hectares of rural area close to Ostrava city in the Czech Republic. The main object of interest was the Opava river creating a set of meanders in this locality with riversides that are covered by fragmented vegetation including bushes and trees up to twenty meters of height. The length of photographed stream was approximately 700 meters. The influence and impact of vegetation on visibility analyses in high detailed large-scale surface model analyses is the articles main research topic.

\section{DATA}

A low-cost Unmanned Air Vehicle (UAV) Phantom 3 Advanced was used for data collection. This mini quadrocopter is standardly equipped with a built-in 12 MPix RGB camera. Flight height was set to 90 meters and orthogonal images were obtained with a $90 \%$ overlapping both in longitudinal and transverse directions. Such a high overlapping together with a selection of windless day and additional collection of oblique images from much lower flight heights were vital for a reasonable reconstruction of existing vegetation in digital surface model (DSM). Total flight time didn't exceed thirty minutes which in relation to covered surface area of 15 hectares shows the effectiveness of UAV in collecting spatial data.

A set of 345 images from UAV entered the processing in the Agisoft Photoscan software (version 1.3.2). The individual photos were aligned and lately a $3 \mathrm{~d}$ point dense cloud was derived using the Structure from Motion (SfM) technique. Its final version consisted of 59 million of points (Fig. 1).
Since the UAV is equipped with a GPS receiver, images from it were geotagged with WGS-84 coordinates. Processing in Agisoft Photoscan was done in this coordinate system, however the final $3 \mathrm{~d}$ dense point cloud was exported in the Czech national coordinate system S-JTSK Krovak East-North (EPSG:5514) for following steps. A standard georeferencing process via ground control points could not be applied since no GCPs were established for this test campaign. In the next step, the $3 \mathrm{~d}$ point cloud was firstly reduced in the Cloud Compare software and lately used for a generation of digital surface model (DSM) suitable for studied visibility analysis. The area on which the research was conducted is visible in Fig.2.

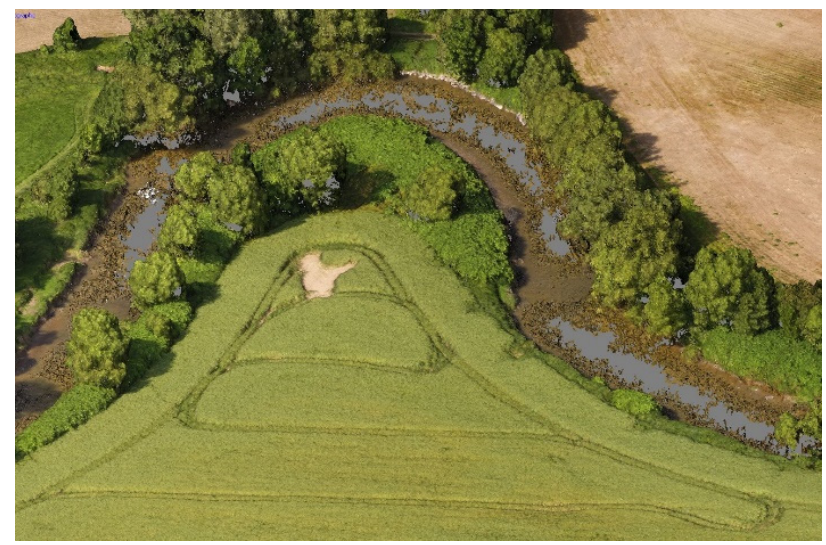

Figure 1. Example of point cloud of the area of interest.

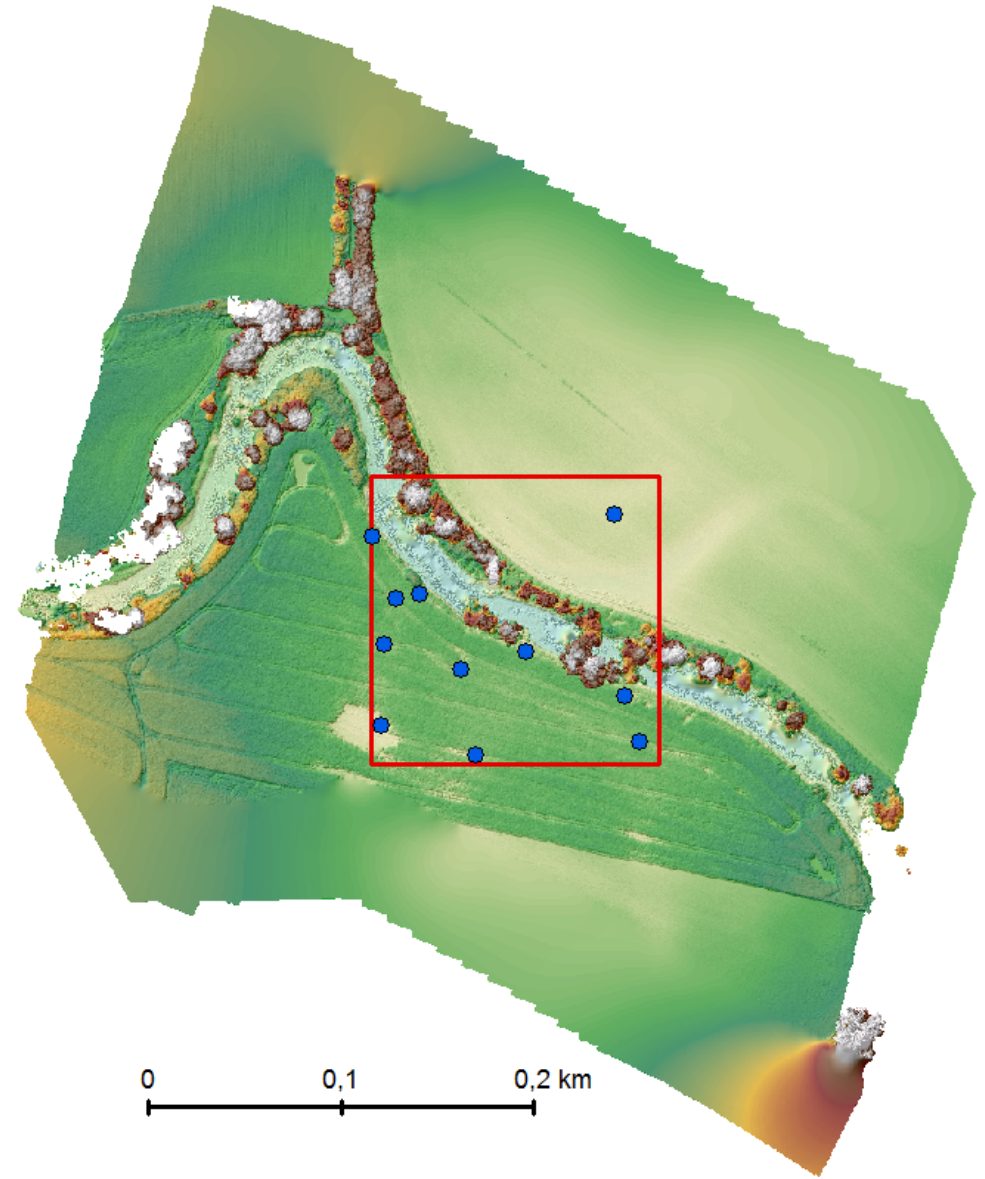

- viewpoints

area of interest

meters above sea level

209.37

Figure 2. Area of the research - river Opava near the Ostrava city (Czech Republic). 
In the dataset the area of interest of size $150 \times 150$ meters (2.25 hectares) was selected for visibility analyses (Fig. 2). The area of interest consists of the river, vegetation along the river (trees and bushes) and two fields. The field north of the river was without vegetation in the time when the data was collected, the field south of the river was overgrown by oilseed rape. In the area of interest, 11 points for visibility analyses were selected. Ten of them located in the southern field, the remaining point is located in the northern field. Points in the southern field are located in places where the vegetation is either trampled or not grown at all.

\section{RESULTS}

The Boolean visibility was calculated for each selected point. Based on these viewsheds the multiple viewshed for set of points can be calculated. The multiple viewshed describes from how many points the area is visible, this type of visibility can be used to identify most prominent features of the surface for the given set of observing points. Besides Boolean viewshed two extended viewsheds were calculated - Global Horizon Viewshed (Fisher, 1996a) and Viewing angle difference to a local horizon (Caha and Rášová, 2015). The global horizon viewsheds from a set of points can be used to determine multiple global horizon viewshed which identifies the most prominent global horizons for the observation points. All the visibility analyses were calculated for an observer with a height of 1.7 meter. This case study focuses on influence of existing vegetation on visibility analyses results in case of detailed and precise surface model.

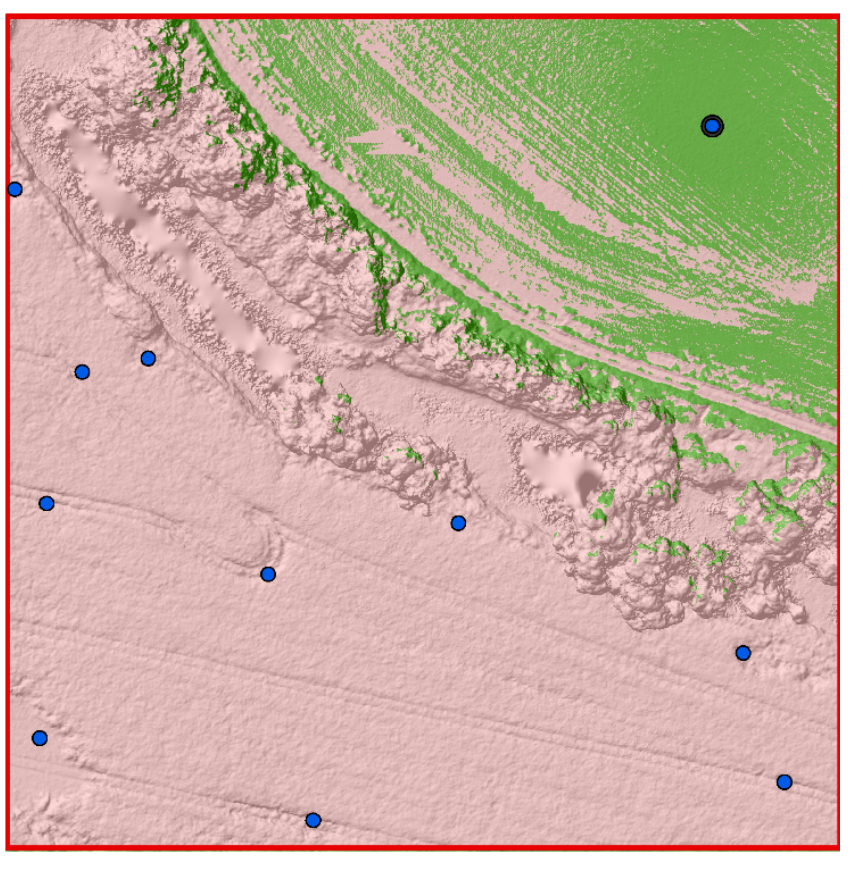

0 50 $100 \mathrm{~m}$
- analyzed viewpoint
- viewpoints

area of interest

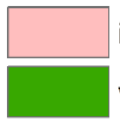

invisible part of surface visible part of surface
Figure 3. Visibility from point north of the river.

The visibility in the area of interest was studied from eleven points. The one located in northeast is located on bare ground with no significant vegetation closely around the point. Due to the nature of its surroundings the visibility is relatively simple with vegetation near the river, on the same bank as the point, acting as the most important barrier (Fig. 3).
The rest of the viewpoints is located on the field south of the river. The points are located in spots without significant vegetation in otherwise grown field of oilseed rape. Because the vegetation along the river is not as distinctive on the southern bank as on the northern part the visibility is more complex and offers view on the vegetation on the opposite bank but unfortunately not behind it. From the ten points located in this part of the area of interest the multiple viewshed is constructed and visualized in Fig. 5. The figure shows that the trees on the northern bank of the Opava river are the most prominent elements of this area visibility-wise. The visibility in those cases is clearly related to the height of the trees which is higher than 15 meters. The trees form a natural visibility barrier in the area of interest. This result is clearly visible not only from the Fig. 5 but also from Fig. 4 which shows multiple global horizon viewshed. Fig. 4 shows only global horizons located in the centre of the area. The algorithm for global horizon viewshed identifies horizons also along edges of selected area, however, such global horizons are not very credible as there is not enough data for their proper identification.

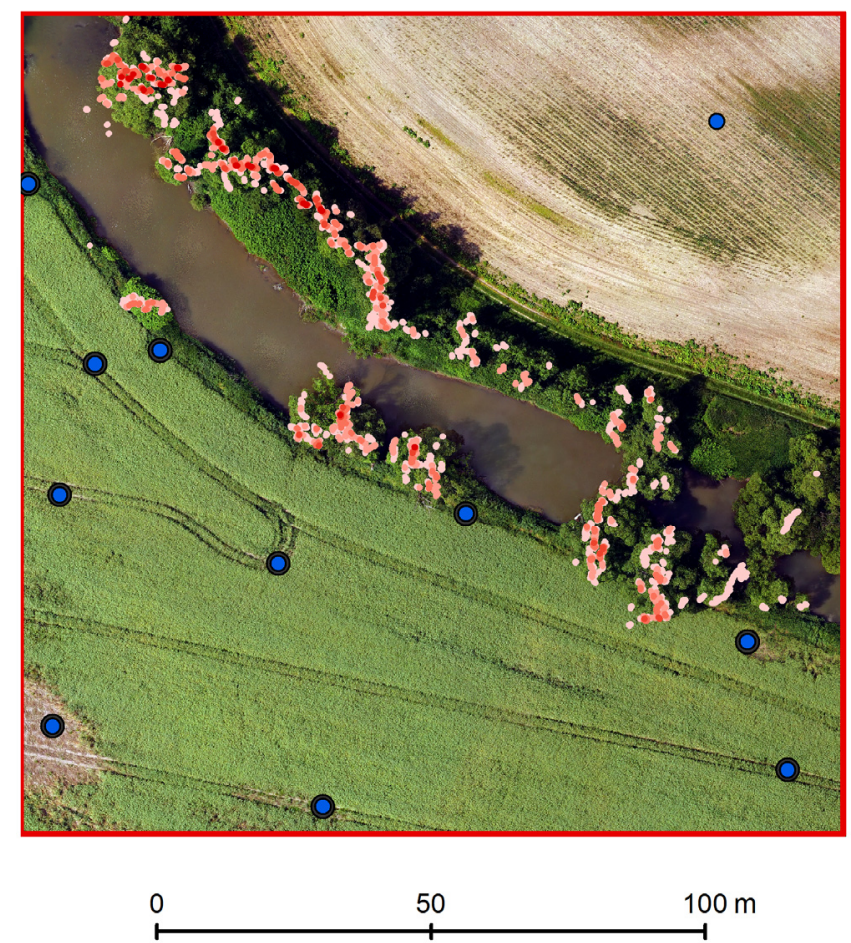

- analyzed viewpoint

number of points from which the location is global horizon

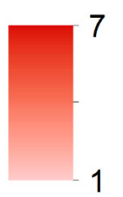

Figure 4. Multiple global horizon viewshed for the points in southern part of the area of interest.

The detail of the model (spatial resolution $7.5 \times 7.5$ centimetres) allows exact identification of a single tree or a bush, sometimes even specific branch of the plant, which is visible or which acts as barrier for visibility. The fact that it is possible to identify the barriers with such precision has great impact on both landscape and urban planning. 


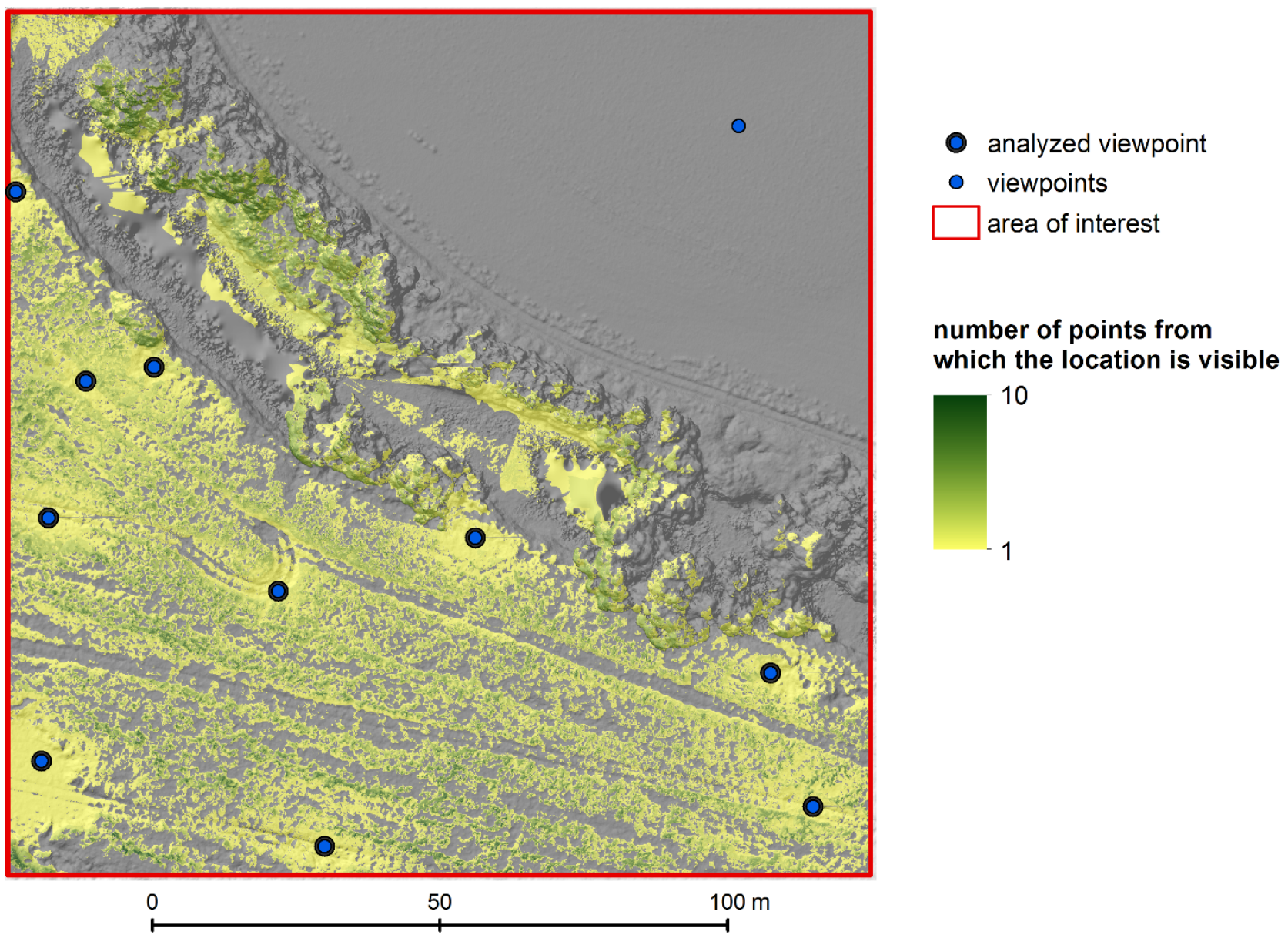

Figure 5. Multiple viewshed from the points in southern part of the area of interest.

Figs. 6 and 7 shows the extended viewshed - angle difference to local horizon (Caha and Rášová, 2015) - calculated from the marked point. The outcome of this analysis highlights the areas that rise significantly over a local horizon (higher values of extended viewshed) from areas that are just barely above the local horizon (values of the extended viewshed close to 0 ). The results show that the vegetation, especially trees, rises relative high above the local horizons. It should be noted that the highest values of this extended viewshed (values higher than 45 degrees) are usually found in small area around the observer, where there are no local horizons present and the value is equal to viewing angle.

All the outcomes show the importance of vegetation in local visibility analysis in unbuilt areas. The amount of detail of the surface allows detailed evaluation of what are the most important features in the area with respect to the visibility.

\section{DISCUSSION AND FUTURE WORK}

This research servers as a pilot study of utilization of large scale surface models for visibility analysis. The data provided by UAVs allows visibility analyses to be easily performed in previously unavailable detail. This brings new possibilities but it also possesses several challenges which will be discussed in this chapter.

The most significant improvement in a form of reached spatial resolution also turns out to be the major challenge while performing visibility analyses. In the case study the spatial resolution of the created digital surface model is $7.5 \times 7.5$ centimetres, which allows to capture considerable details but on the other hand it produces rather large datasets. The visibility analyses are known to be rather demanding in terms of memory space and computational time (van Kreveld, 1996; Haverkort et al., 2009) and even though that there have been significant improves in these research topics lately (Ferreira et al., 2014; Tabik et al., 2015) there are still issues to be solved, especially when it comes to optimization of extended viewshed determination. The problem is that most of the research regarding optimization of computational efficiency is focused on a classic Boolean viewshed and while some findings can be applied to the processes of extended viewsheds determination there are also issues that need to be solved specifically for extended viewsheds. This could open a completely new area of research focused on optimization of algorithms for extended viewsheds determination.

Even though, that the previously mentioned challenges regarding time and computational requirements of the visibility analyses might seem like an issue there are ways of overcoming them. One of them is calculation of viewsheds for only part of the surface, which is mentioned as optimization technique by Haverkort et al. (2009), or by determining the extended viewsheds on point to point basis, as shown by Caha (2017). Both these approaches significantly lower the demands and provide the valuable outcomes.

With the surface being very precise and detailed it would be highly relevant for studies of visibility with cities such are 
presented by Czyńska and Rubinowicz (2017), Rubinowicz and Czyńska (2015), Wrózynski et al. (2016) or Rød and van der Meer (2009). All these studies focus on visibility analysis and skyline protection with respect towards specific buildings (structures) or panoramas which usually require data on regional scale. However, there are elements (for example statues, artistic installations, specific vistas etc.) which are significantly smaller than buildings, they are visible only from close neighbourhood and relatively small changes in the environment of the city, such as growth of vegetation or construction of a pole, might negatively affect their visibility. To assess visibility and potential change in visibility of these elements the visibility analyses need to be performed in local scale on large-scale models that are able to capture all the necessary details.

As mentioned and referenced previously, some studies showed that it is possible to use data scanned from UAV to construct real 3D data. Such data allows modelling differences between spatial extent of trunk and crow of the tree. 3D model and voxel approach allow even more detailed analysis of the visibility, as shown by Chmielewski and Tompalski (2017). Studies were visibility through linear vegetation are considered, e.g. our case study or study of Wrózynski et al. (2016), would benefit from this approach as it is likely that the linear vegetation would offer some vistas that we are unable to model with current $2.5 \mathrm{D}$ surface model. Unfortunately, extended viewsheds were not yet used in visibility analyses calculated on voxel surface model. This topic should be one of the possible directions for future research.

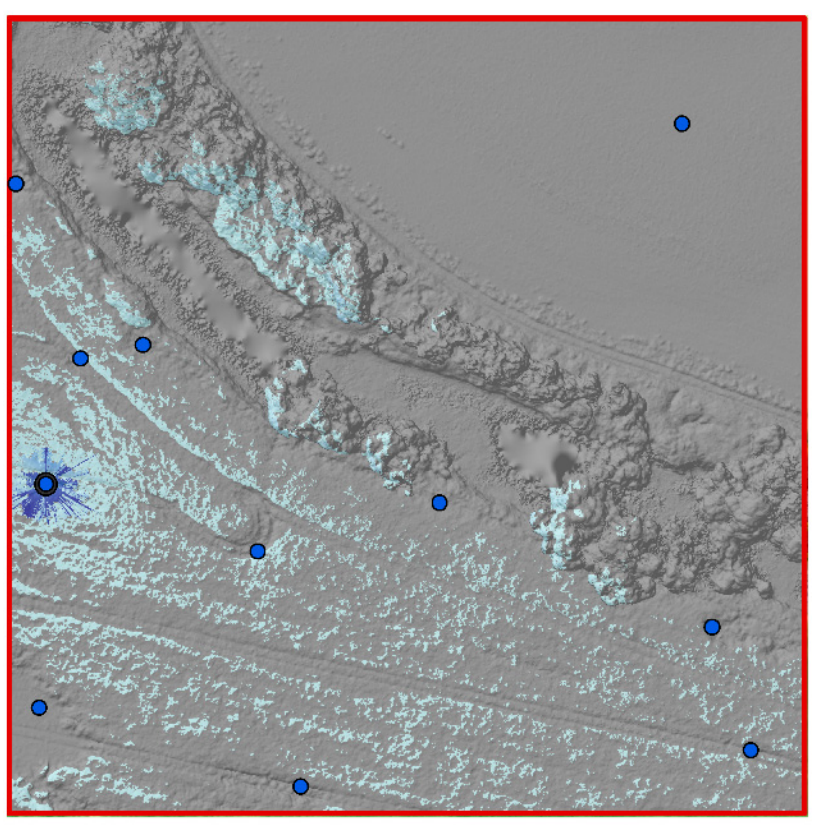

0

50 $100 \mathrm{~m}$

analyzed viewpoint

- viewpoints area of interest

angle difference to local horizon

(degrees)

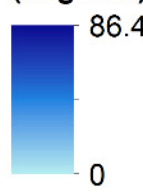

Figure 6. Extended viewshed - angle difference to the local horizon.

\section{CONCLUSION}

The presented research is the first step in utilization of large scale surface models for visibility analysis. The visibility is usually studied on larger areas, e.g. visibility of high-rise building in the context of the city, but there are applications where local visibility analyses are rather important topic. These applications may span from visibility of statues and other smaller monuments in the spatial context of street or square, to visibility of traffic and other signs from its surroundings, other applications may include planning of military operations or finding optimal observation points. The results can also be used in landscape and urban planning where the user might be interested in finding and preserving important vistas, tourist route planning, evaluation of viewpoints, etc. In all these cases the detailed surface models significantly improve the outcomes of the analysis by making it much more precise. The surface data can also be used for so called what-if modelling, comparison of different scenarios of how will an intervention, which might be a planting or felling of vegetation or various construction works, affect the visibility from a viewpoint or visibility of specific target points. Detailed surface models allow an assessment of the impact of such interventions on the visibility before it actually occurs. However, for such analysis it is necessary to have up-to-date detailed surface data and the utilization of UAVs allows fast, cheap and precise acquisition of data that can be updated relatively often as vegetation or urban conditions can change significantly. Overall, the topic of utilization of detailed surface data in viewshed analyses seems as an interesting topic that provides several relatively new and important research areas.

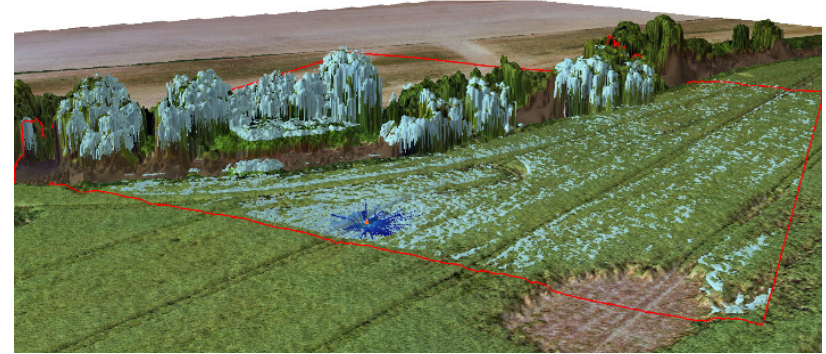

Figure 7. 3D visualization of extended viewshed - angle difference to the local horizon.

\section{ACKNOWLEDGEMENTS}

The study was supported by project IGA FRDIS Mendel University in Brno No. 022/2017 "Visibility analysis of high-rise buildings as tool for urban and landscape planning" (FRRMS_IGA_2017/022) and by project no. SP2017/25 of the Student's Grant Competition at VŠB-Technical University of Ostrava

\section{REFERENCES}

Caha, J., 2017. Hodnocení viditelnosti plánovaných výškových budov Prague Eye Towers. Geogr. časopis 69, pp. 75-92.

Caha, J. and Rášová, A., 2015. Line-of-Sight Derived Indices: Viewing Angle Difference to a Local Horizon and the Difference of Viewing Angle and the Slope of Line of Sight. Rủžičková, K., Inspektor, T. (Eds.), Surface Models for Geosciences SE - 6. Springer International Publishing, Cham, pp. 61-72. 
Czyńska, K. and Rubinowicz, P., 2017. Sky Tower impact on the landscape of Wrocław - analysing based on the VIS method. Architectus 2, pp. 87-98.

Chmielewski, S. and Tompalski, P., 2017. Estimating outdoor advertising media visibility with voxel-based approach. Appl. Geogr. 87, pp. 1-13.

Dandois, J.P. and Ellis, E.C., 2013. High spatial resolution threedimensional mapping of vegetation spectral dynamics using computer vision. Remote Sensing of Environment, 136, pp. 259276.

Ferreira, C.R., Andrade, M.V.A., Magalhães, S.V.G., Franklin, W.R. and Pena, G.C., 2014. A Parallel Algorithm for Viewshed Computation on Grid Terrains. Journal of Information and Data Management, 5(2), p. 171.

Fisher, P.F., 1996a. Reconsideration of the viewshed function in terrain modelling. Geographical Systems, 3, pp. 33-58.

Fisher, P.F., 1996b. Extending the Applicability of Viewsheds in Landscape Planning. Photogramm. Eng. Remote Sensing, 62, $1297-1302$

Haverkort, H., Toma, L. and Zhuang, Y., 2009. Computing Visibility on Terrains in External Memory. ACM J. Exp. Algorithmics 13.

Klouček, T., Lagner, O. and Š́mová, P., 2015. How does data accuracy influence the reliability of digital viewshed models? A case study with wind turbines. Appl. Geogr. 64, pp. 46-54.

Lee, J., 1991. Analyses of visibility sites on topographic surface. International Journal of Geographical Information System, 5(4), pp. 413-429.

Maloy, M.A. and Dean, D.J., 2001. An Accuracy Assessment of Various GIS-Based Viewshed Delineation Techniques. Photogramm. Eng. Remote Sens. 67, pp. 1293-1298.
Remondino, F., Barazzetti, L., Nex, F., Scaioni, M. and Sarazzi, D., 2011. UAV photogrammetry for mapping and 3d modeling Current status and future perspectives. International Archives of the Photogrammetry, Remote Sensing and Spatial Information Sciences, 38(1), pp. 25-31.

Rød, J.K. and van der Meer, D., 2009. Visibility and dominance analysis: Assessing a high-rise building project in Trondheim. Environment and Planning B: Planning and Design, 36(4), pp. 698-710

Rubinowicz, P. and Czyńska, K., 2015. Study of city landscape heritage using Lidar data and 3D-city models. The International Archives of the Photogrammetry, Remote Sensing and Spatial Information Sciences, 40(7), pp. 1395-1402.

Tabik, S., Cervilla, A.R., Zapata, E. and Romero, L.F., 2015. Efficient Data Structure and Highly Scalable Algorithm for Total-Viewshed Computation. IEEE Journal of Selected Topics in Applied Earth Observations and Remote Sensing, 8(1), pp. 304-310.

Tomaštík, J., Mokroš, M., Saloš, S., Chudỳ, F. and Tunák, D., 2017. Accuracy of photogrammetric UAV-based point clouds under conditions of partially-open forest canopy. Forests, 8(5), p. 151

van Kreveld, M., 1996. Variations on Sweep Algorithms: efficient computation of extended viewsheds and class intervals. Utrecht University: Information and Computing Sciences, Utrecht.

Wrózynski, R., Sojka, M. and Pyszny, K., 2016. The application of GIS and 3D graphic software to visual impact assessment of wind turbines. Renewable Energy 96, pp. 625-635. 